\title{
GMR
}

\section{Identification of Magnolia wufengensis (Magnoliaceae) cultivars using phenotypic traits, SSR and SRAP markers: insights into breeding and conservation}

L. Wang ${ }^{1 *}$, A.H. Xiao ${ }^{1 *}$, L.Y. Ma ${ }^{1}$, F.J. Chen ${ }^{2}$, Z.Y. Sang ${ }^{3}$ and J. Duan ${ }^{1}$

${ }^{1}$ Key Laboratory for Silviculture and Conservation of the Ministry of Education, Beijing Forestry University, Beijing, China

${ }^{2}$ Biotechnology Research Center, China Three Gorges University,

Yichang, Hubei Province, China

${ }^{3}$ Forestry Bureau of Wufeng County, Hubei Province, China

*These authors contributed equally to this study.

Corresponding author: L.Y. Ma

E-mail: maluyi@bjfu.edu.cn

Genet. Mol. Res. 16 (1): gmr16019473

Received October 25, 2016

Accepted December 19, 2016

Published February 23, 2017

DOI http://dx.doi.org/10.4238/gmr16019473

Copyright (C) 2017 The Authors. This is an open-access article distributed under the terms of the Creative Commons Attribution ShareAlike (CC BY-SA) 4.0 License.

\begin{abstract}
A combination of phenotypic characterization and molecular markers may provide reliable information on new plant varieties and elucidate the conservation status of rare species. Five newly developed Magnolia wufengensis cultivars, an endangered plant species endemic to Hubei Province, China, possess more distinctive phenotypes than common Magnolia cultivars. With reference to a wild species population of $M$. wufengensis and a population of Magnolia denudata, morphological traits of flower organs, simple sequence repeat (SSR), and sequence-related amplified polymorphism (SRAP) markers were used. In the morphological study, six traits of floral organs were investigated and their relationships were analyzed between cultivars. In the genetic study, 9 SSR primer pairs and 10 SRAP primer
\end{abstract}


combinations were screened. The five cultivars maintained a high level of genetic diversity. Genetic diversity of each $M$. wufengensis cultivar was much lower than that of the wild population, but was slightly higher than that of the M. denudata population. Analysis of molecular variance (AMOVA) revealed that genetic variation among populations was $20 \%$ (SRAP) and 30\% (SSR), which showed a high degree of genetic differentiation among populations of the five cultivars. The dendrograms illustrated a clear separation between $M$. wufengensis populations and outer species, and identified two major groups among cultivars. Correlation analysis indicated a good fit between the two marker systems, but a relatively low fit between morphological and genetic traits (SRAP: $r=0.60$, SSR: $r=0.52$ ). These findings provide reliable references for the application of these molecular markers in the breeding and conservation of $M$. wufengensis.

Key words: Cultivars; Genetic diversity; Magnoliaceae; Magnolia wufengensis; SSR; SRAP

\section{INTRODUCTION}

Magnolia wufengensis is an endangered, rare species belonging to the family Magnoliaceae, endemic to Hubei Province, China, and renowned for its showy flowers with variable tepal number, color, and shape (Ma et al., 2006). Since first being observed in 2004, germplasm with different characters has been screened and collected. Five germplasms with eminent properties have been artificially propagated by large-scale grafting and officially registered as new cultivars in the State Forestry Administration Office for the Protection of New Varieties of Plants in China. Over years of experiments on test grounds, the survival rate of grafting seedlings has reached over $90 \%$. As a result of continuous research on cold resistance, the five cultivars can now be suitably propagated in Northern China, Central China, Southwest China, and South China, which represent different plant cold hardiness zones (Yang et al., 2015a,b,c). Because of the high variation in morphological traits, such as tepal number and color, these plants are key ornamental trees for both mountain and urban landscapes, and they have great importance in economic application and academic research. Although previous studies have provided preliminary data to identify the species of $M$. wufengensis (He et al., 2007; Chen et al., 2014), little is known about the genetic diversity and structure of cultivars. In addition, due to the large number of breeding activities and a rapid increase in the number of seedlings, a mix of cultivars and seedlings with unclear genetic background has occurred frequently, which negatively affects species conservation as well as further academic research. Therefore, further studies are needed to classify, introduce, domesticate, and propagate these cultivars and finally make them commercially available. More importantly, because the cultivars possess precious traits of $M$. wufengensis, breeding of these cultivars is advantageous to the conservation of the species.

As one of the most distinguishing characteristics of M. wufengensis, the morphological phenotypes are essential for scientific planting schemes and for cultivar selection (Mratinić et al., 2014). Investigation on the morphological traits of different organs has revealed high variation in the phenotypes of $M$. wufengensis (He et al., 2007). Furthermore, these traits

Genetics and Molecular Research 16 (1): gmr16019473 
will eventually form the basic classification reference for cultivar screening. Additionally, studying morphological traits of different cultivars would also permit the identification of eminent phenotypes with stable inheritance for genetic functional research (Abid et al., 2015). Based on a study that investigated how flower color was affected by the structure of flower petals, a theoretical basis for safflower formation and origin was sought (He et al., 2010).

Genetic relationships among cultivars are informative for scientific researchers, breeders, and public policy makers. Although morphological traits can be measured easily, they are subject to numerous constraints and are particularly dependent on the environment. Nevertheless, molecular markers can provide more accurate information and can be used to detect variation among individuals, populations, and species. Numerous molecular markers have been used widely for cultivar identification. Chen et al. (2014a) used simple sequence repeat (SSR) markers to identify walnut cultivars. Guo et al. (2012) applied sequence-related amplified polymorphism (SRAP) markers to cultivated grape varieties. Inter-simple sequence repeat (ISSR) and amplified fragment length polymorphism (AFLP) markers were detected and used to study hazelnut cultivars (Sabir et al., 2014), random amplified polymorphic DNA (RAPD) was developed for the study of crop plants (Souframanien and Gopalakrishna, 2004), and Tang et al. (2013) used single nucleotide polymorphisms (SNPs) to assess tulip cultivars. SSR and SRAP markers proved to be highly polymorphic for identifying cultivated varieties (György et al., 2016).

Molecular markers used for the evaluation of genetic diversity have both advantages and disadvantages. Compared to other marker systems, the SRAP technique is simple, reliable, of moderate throughput, and does not require any prior knowledge of the genomic sequence (Li and Quiros, 2001). Based on a study on buffalo grass, the SRAP system was found to be more powerful than ISSR and RAPD markers (Budak et al., 2004). Microsatellites are hypervariable single-locus markers, and the use of modern bioinformatics, such as expressed sequence tag (EST) databases, have made them easier to access. SSR markers have many advantages over other markers, such as their codominance, high reproducibility, relatively high levels of polymorphism, and abundant distribution in genomes. In studies on soybean germplasm, SSR markers have been found to have higher heterozygosity than RFLP, RAPD, and AFLP markers (Powell et al., 1996). The widely used SSR and SRAP marker systems have been utilized for the genetic study of $M$. wufengensis cultivars.

The objectives of our study were to 1) provide valuable information for proper cultivar selection by comparing morphological traits and developing practical molecular markers for five new M. wufengensis cultivars; 2) evaluate the level of genetic diversity and analyze the genetic differentiation of cultivars through SSR and SRAP markers; 3) propose conservation measures and breeding strategies for $M$. wufengensis based on its genetic variation.

\section{MATERIAL AND METHODS}

\section{Plant material and DNA extraction}

Five extant cultivars of M. wufengensis in Hubei Province, China, were selected for our study, namely 'Jiaohong No.1' (JH1), 'Jiaohong No.2' (JH2), 'Jiaoju' (JJ), 'Jiaozi' (JZ), and 'Jiaoyan' (JY). A range of 30 to 36 individual plants of each population were sampled (Table 1). Samples of wild M. wufengensis species and one other species of Magnoliaceae, Magnolia denudata, were used as references. Three young leaves of each individual plant

Genetics and Molecular Research 16 (1): gmr16019473 
were collected for DNA extraction and quickly frozen in liquid nitrogen before being stored at $-80^{\circ} \mathrm{C}$ until use (Table 1 ).

Table 1. Information on the populations used in this study.
\begin{tabular}{l|c|c|l|c}
\hline Sample name & Variety rights No. & Date of variety rights certificate & Sample site location & Sample size \\
\hline JH1 & 20120073 & 2012.07 .31 & Wufeng, Hubei Province & 36 \\
\hline JH2 & 20140050 & 2014.06 .27 & Botanical Garden of Three Gorges, Hubei Province & 34 \\
\hline JJ & 20140042 & 2014.06 .27 & Wuhan, Hubei Province & 31 \\
\hline JZ & 20140043 & 2014.06 .27 & Wuhan, Hubei Province & 30 \\
\hline JY & 20140044 & 2014.06 .27 & Wuhan, Hubei Province & 30 \\
\hline WS & - & - & Wufeng, Hubei Province & 16 \\
\hline MD & - & - & Beijing Botanical Garden, Beijing & 12 \\
\hline
\end{tabular}

M. wufengensis cultivars: JH1 = 'Jiaohong No.1', JH2 = 'Jiaohong No.2', JJ = 'Jiaoju', JZ = 'Jiaozi', JY = 'Jiaoyan'; reference populations: $\mathrm{WS}=M$. wufengensis wild species, $\mathrm{MD}=M$. denudata .

Genomic DNA of single individuals was extracted using TaKaRa MiniBEST Plant Genomic DNA Extraction Kit (Dalian, China). The concentration and quality of DNA samples were checked using a Thermo Scientific NanoDrop 2000 spectrophotometer (Massachusetts, USA) to ensure both $260 / 280$ and $260 / 230$ values were no less than 1.8 . The DNA samples were diluted to approximately $10 \mathrm{ng} / \mu \mathrm{L}$ in $0.1 \%$ TE buffer.

\section{Phenotypic measurements}

Six distinguished characters of flower organs were evaluated and compared, including tepal color, tepal number, tepal width, tepal length, style length, and stamen length. The three outermost tepals of one flower were tested.

Tepal color was defined using the Royal Horticultural Society Color Chart (RHSCC) (http://www.azaleas.org/index.pl/rhsmacfan2.html), and total anthocyanin content was measured using the high performance liquid chromatography (HPLC) method. Tepals $(0.5$ g) were ground in $2 \mathrm{~mL}$ acidic methanol $\left(0.1 \%\right.$ hydrochloric acid) at $4^{\circ} \mathrm{C}$, then centrifuged at $10,000 \mathrm{~g}$ for $10 \mathrm{~min}$ at $4^{\circ} \mathrm{C}$. The supernatant was passed through a $0.22-\mathrm{mm}$ syringe filter before HPLC analysis. Anthocyanin was investigated on an Agilent 1260 HPLC equipped with a VWD detector (Agilent Technology, USA), as described by Fan et al. (2012). Total anthocyanin content was measured semi-quantitatively from a simple linear regression using cyanidin-3-O-glucoside (Cy3G) (Sigma-Aldrich, USA) as a standard at $520 \mathrm{~nm}$.

\section{SSR and SRAP marker development}

Overall, 176 pairs of SSR primers originally from EST-SSRs of Liriodendron tulipifera were collected (Xu et al., 2010), among which 32 pairs reported to have implications for the major phylogeny of Magnoliaceae, were screened in our study (Yang et al., 2012a). Primers that did not generate any amplification products, or that produced a number of faint bands due to non-specific amplifications, were excluded. Eventually, nine pairs that produced a single prominent band were selected to identify cultivars (Table 2).

All SSR primers were synthesized by the Sangon Biotech (Shanghai) Co, Ltd. Among the overall 48 SRAP primer pair combinations (em1-em6 x me1-me8), 10 primer pair combinations, including em1- me1, em1- me3, em1- me5, em2- me5, em4- me1, em5me3, em5- me4, em5- me5, em5- me8, and em6- me5 were valid for M. wufengensis (Chen 
et al., 2014). All SRAP primers were synthesized by the Sangon Biotech (Shanghai) Co., Ltd. (Table 3).

Table 2. Characteristics of nine expressed sequence tagged simple sequence repeat (EST-SSR) loci.

\begin{tabular}{l|c|c|l|l|l|l}
\hline Locus & Size $(\mathrm{bp})$ & Repeat motif & \multicolumn{4}{|c}{ Primer sequences $\left(5^{\prime}-3^{\prime}\right)$} \\
\hline LT013 & 271 & $(\mathrm{GA})_{17}$ & $\mathrm{~F}$ & CATGTCTGGTGGAAGAGAAT & $\mathrm{R}$ & CCATGAGAAGAGGATGAAAC \\
\hline LT023 & 161 & $($ TTCGTC $)$ & F & TGATAGATATGGAGGGTGGA & R & TGAAGACGAGTTCCCAGTAT \\
\hline LT078 & 228 & $(\text { AACA })_{5}$ & F & ACTGGGCCGTTTATACTTTT & R & TGACCTTTCCCTTATTCTCA \\
\hline LT083 & 206 & $(\text { ACAACC })_{4}$ & F & ATTACGCAGCTTCCCTTAC & R & GGAGTTCTGGTATGGTTGAG \\
\hline LT087 & 294 & $(\text { ACTCGG })_{4}$ & F & CATCGCCTCACTAACACTC & R & GACATCATCCTCCATCTCC \\
\hline LT092 & 229 & $(\text { GGAGCC })_{4}$ & F & GGGGTTTTGCTTAATGTGA & R & CATTCCCTACCTCCTTCTCT \\
\hline LT115 & 129 & $(\text { TCA })_{9}$ & F & CTCTCATTCCGACCTTCATA & R & ACTTTTCCTGCAACTACTGC \\
\hline LT149 & 125 & $(\text { CTT })_{6}$ & F & GTGAAGACCAAGGAAAATGA & R & GGAAAAGAGAGGAGGAATGT \\
\hline LT164 & 222 & $(\text { CTT })_{7}$ & F & AGCTGCCAAGACCTACAAC & R & CCTCTTCAGGTTCCACATTA \\
\hline
\end{tabular}

Table 3. Sequences of 10 sequence-related amplified polymorphism (SRAP) primers.

\begin{tabular}{l|l|l|l}
\hline Primers (F) & Sequence $\left(5^{\prime}-3^{\prime}\right)$ & Primers $(\mathrm{R})$ & Sequence $\left(5^{\prime}-3^{\prime}\right)$ \\
\hline em1 & GACTGCGTACGAATTAAT & me 1 & TGAGTCCAAACCGGATA \\
\hline em2 & GACTGCGTACGAATTTGC & me3 & TGAGTCCAAACCGGAAT \\
\hline em4 & GACTGCGTACGAATTTGA & me 4 & TGAGTCCAAACCGGACC \\
\hline em5 & GACTGCGTACGAATTAAC & me5 & GAGTCCAAACCGGAAG \\
\hline em5 & GACTGCGTACGAATTGCA & me8 & TGAGTCCAAACCGGTGC \\
\hline
\end{tabular}

PCR amplification was carried out in a $10-\mu \mathrm{L}$ reaction system, containing $1 \mu \mathrm{L} 10 \mathrm{ng} /$ $\mu \mathrm{L}$ genomic DNA, $0.5 \mu \mathrm{L}(10 \mathrm{mmoL})$ each primer, $5 \mu \mathrm{L} 2 \mathrm{X}$ Taq premix (Takara, Japan), and 3 $\mu \mathrm{L} \mathrm{ddH}_{2} \mathrm{O}$. Amplification reactions were performed by a Bio-rad T100 cycler (Bio-rad, USA). The SSR marker program was as follows: initial denaturation for $5 \mathrm{~min}$ at $94^{\circ} \mathrm{C}, 15$ cycles of $30 \mathrm{~s}$ at $94^{\circ} \mathrm{C}, 30 \mathrm{~s}$ at $60^{\circ} \mathrm{C}$ decreasing to $49.5^{\circ} \mathrm{C}$ by $0.7^{\circ} \mathrm{C}$ per cycle, and $30 \mathrm{~s}$ at $72^{\circ} \mathrm{C}$, followed by 20 cycles of $30 \mathrm{~s}$ at $94^{\circ} \mathrm{C}, 30 \mathrm{~s}$ at $49.5^{\circ} \mathrm{C}$, and $72{ }^{\circ} \mathrm{C}(30 \mathrm{~s})$, followed by $20 \mathrm{~min}$ at $72^{\circ} \mathrm{C}$ (Xu et al., 2010). The SRAP marker program was as follows: initial denaturation for $5 \mathrm{~min}$ at $94^{\circ} \mathrm{C}, 5$ cycles of $1 \mathrm{~min}$ at $94^{\circ} \mathrm{C}, 1 \mathrm{~min}$ at $35^{\circ} \mathrm{C}$, and $1 \mathrm{~min}$ at $72^{\circ} \mathrm{C}$, followed by 35 cycles of $30 \mathrm{~s}$ at $94^{\circ} \mathrm{C}, 1 \mathrm{~min}$ at $49.5^{\circ} \mathrm{C}$, and $1 \mathrm{~min}$ at $72^{\circ} \mathrm{C}$ ( $\mathrm{Li}$ and Quiros, 2001). Native-PAGE (8\%) were undertaken utilizing silver-staining for SSR band detection, and a DL500 DNA marker (Takara, JP) was used to calculate the size of the bands.

\section{Data analysis}

Bands with the same weight and length were considered as alleles. Weak or ambiguous bands were not counted. SSR analyses were undertaken in a co-dominant way, while SRAP analyses were calculated through dominant ways. For parallel comparison between the two markers, only indices that could be conducted by each of the two statistical methods were studied. These data were compiled into a matrix for each primer, which was adjoined differently for the analyses.

The mean effective number of alleles, the number and percentage of polymorphic loci $(99 \%$ criterion), the mean expected heterozygosity, and Shannon index were calculated via POPGENE32 to describe genetic diversity. Based on the data of genetic distances and dissimilarity coefficients, unweighted pair-group method using arithmetic averages (UPGMA) analysis and Mantel tests were driven by NTSYS-pc version 2.1. Associations between genetic diversity parameters were evaluated by EXCEL 2016. Results of the $t$-test were analyzed using SPSS version 20.0. AMOVA analysis was driven by GenAlEx 6.5. 


\section{RESULTS}

\section{Morphological phenotype identification}

Apparent and considerable phenotypic differences were observed among the five M. wufengensis cultivars (Figure 1A-O). The descriptive statistics for the selections of floral organs studied are provided in Table 4.

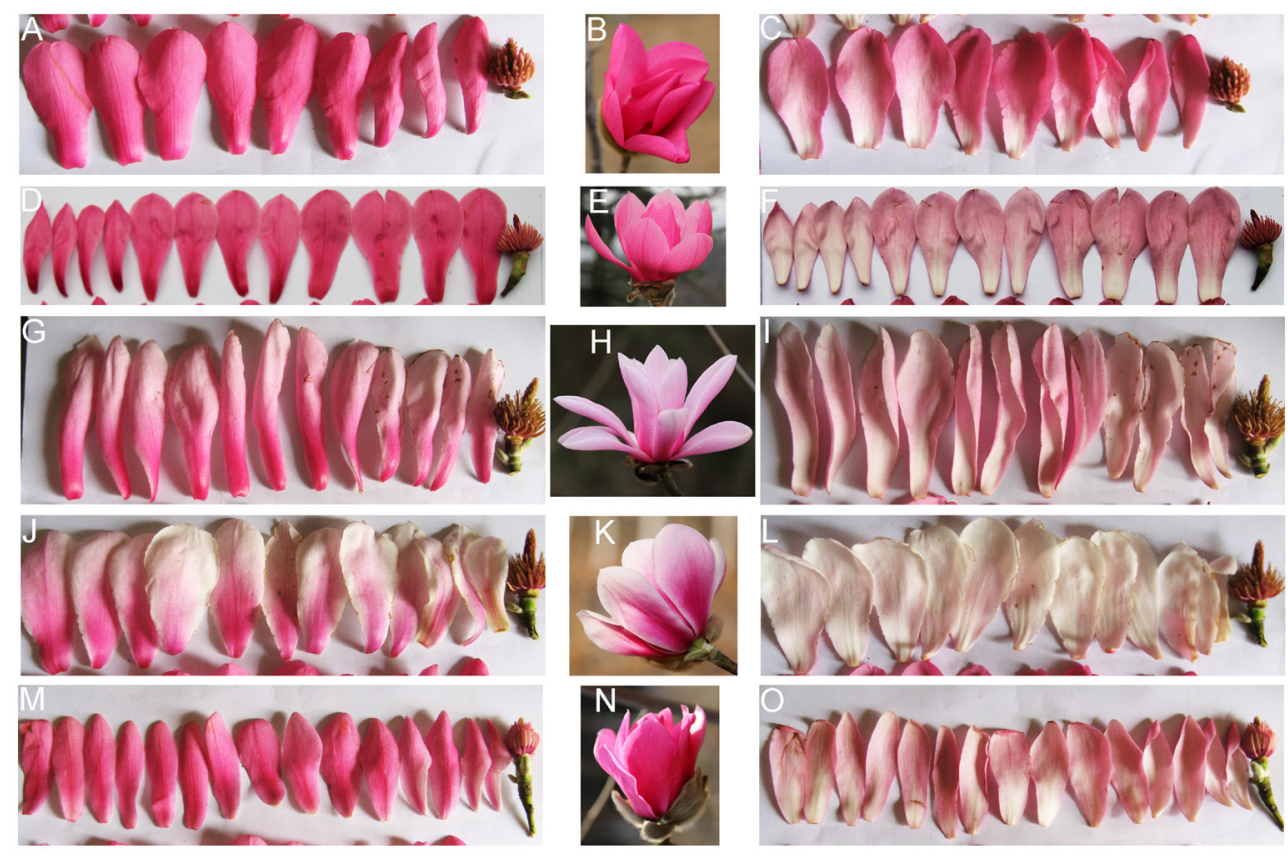

Figure 1. Floral phenotypes of five Magnolia wufengensis cultivars. A. B. C. the abaxial tepals, appearance of flower, adaxial tepals of 'Jiaohong No.1'; D. E. F. the abaxial tepals, appearance of flower, adaxial tepals of 'Jiaohong No.2'; G. H. I. the abaxial tepals, appearance of flower, adaxial tepals of 'Jiaoju'; J. K. L. the abaxial tepals, appearance of flower, adaxial tepals of 'Jiaozi'; M. N. O. the abaxial tepals, appearance of flower, adaxial tepals of 'Jiaoyan'.

Table 4. Phenotypic comparisons of different floral organs among different populations.

\begin{tabular}{l|c|c|c|c|c}
\hline & Tepal number & Tepal width $(\mathrm{cm})$ & Tepal length $(\mathrm{cm})$ & Style length $(\mathrm{cm})$ & Stamen length $(\mathrm{mm})$ \\
\hline JH1 & $9.20 \pm 0.63^{\mathrm{c}}$ & $2.75 \pm 0.07^{\mathrm{c}}$ & $7.16 \pm 0.06^{\mathrm{c}}$ & $3.22 \pm 0.06^{\mathrm{c}}$ & $14.03 \pm 0.06^{\mathrm{d}}$ \\
\hline JH2 & $12.20 \pm 0.63^{\mathrm{b}}$ & $2.69 \pm 0.04^{\mathrm{c}}$ & $7.26 \pm 0.03^{\mathrm{c}}$ & $3.19 \pm 0.03^{\mathrm{c}}$ & $14.01 \pm 0.07^{\mathrm{d}}$ \\
\hline JJ & $18.80 \pm 4.66^{\mathrm{a}}$ & $2.38 \pm 0.08^{\mathrm{d}}$ & $7.77 \pm 0.03^{\mathrm{a}}$ & $3.62 \pm 0.03^{\mathrm{b}}$ & $15.46 \pm 0.21^{\mathrm{b}}$ \\
\hline JZ & $12.00 \pm 0.00^{\mathrm{b}}$ & $2.78 \pm 0.11^{\mathrm{c}}$ & $7.44 \pm 0.08^{\mathrm{b}}$ & $3.21 \pm 0.05^{\mathrm{c}}$ & $14.30 \pm 0.07^{\mathrm{c}}$ \\
\hline JY & $13.50 \pm 1.43^{\mathrm{b}}$ & $3.00 \pm 0.06^{\mathrm{b}}$ & $6.69 \pm 0.20^{\mathrm{d}}$ & $3.00 \pm 0.08^{\mathrm{d}}$ & $13.69 \pm 0.06^{\mathrm{e}}$ \\
\hline WS & $13.40 \pm 4.98^{\mathrm{b}}$ & $2.70 \pm 0.27^{\mathrm{c}}$ & $7.25 \pm 0.46^{\mathrm{c}}$ & $3.23 \pm 0.21^{\mathrm{c}}$ & $14.37 \pm 0.74^{\mathrm{c}}$ \\
\hline MD & $9.00 \pm 0.00^{\mathrm{c}}$ & $3.43 \pm 0.12^{\mathrm{a}}$ & $6.18 \pm 0.06^{\mathrm{e}}$ & $3.75 \pm 0.07^{\mathrm{a}}$ & $18.76 \pm 0.45^{\mathrm{a}}$ \\
\hline
\end{tabular}

$\mathrm{JH} 1=$ 'Jiaohong No.1', JH2 = 'Jiaohong No.2', JJ = 'Jiaoju', JZ = 'Jiaozi', JY = 'Jiaoyan'; reference populations: $\mathrm{WS}=M$. wufengensis wild species, $\mathrm{MD}=M$. denudata. Data are reported as means \pm standard deviation $(\mathrm{SD})$. Different letters indicate a significant difference between populations. $\mathrm{P}<0.05$. 
Tepal number ranged from nine to 24 among $M$. wufengensis cultivars. JH1 (Figure $1 \mathrm{~A}-\mathrm{C}$ ) possessed only nine tepals (seldom 11), which was similar to the number observed in the reference population. MD. Other M. wufengensis cultivars, as well as the WS population, had high tepal numbers, and JJ (Figure 1G-I) possessed an average of 18.8 tepals (Table 4 ) and was also high in variety $(\mathrm{SD}=4.66)$.

The shape of tepals varied from obovate to long lanceolate (Figure 1A-O). The cultivar $\mathrm{JJ}$ (Figure 1G-I) exhibited the longest tepal length $(7.77 \pm 0.03)$ and the minimum width (2.38 \pm 0.08 ) (Table 4), which resulted in chrysanthemum-shaped flowers. Compared to the MD, all the tepal shapes of $M$. wufengensis cultivars, as well as WS, were shorter and narrower.

For style and stamen length, JJ was significantly longer than the other cultivars, while both of these two organs were the smallest in JY. Again, the reference population MD possessed a much larger style and stamen than the other M. wufengensis populations (Table 4).

Therefore, five cultivars of $M$. wufengensis were found to have remarkable differences in their floral organ phenotypes, and were clearly distinguished from the outer populations, MD.

\section{Tepal color determination}

Tepal colors among the five cultivars ranged from strong purplish red to pale purplish pink on adaxial and abaxial sides (Figure 1A-O). The cultivar JH1 (Figure 1A, C) presented the most impressive red color on both tepal sides, RHS 67A (strong purplish red) to RHS 67B (vivid purplish red) abaxially and RHS 67B (vivid purplish red) to RHS 67C (deep purplish pink) adaxially. The striking pink flowers of JZ (Figure 1J, L) presented the lightest color, which ranged from RHS 62A (strong purplish pink) to RHS 62B (moderate purplish pink) on the abaxial, side and the color gradually faded from the base to the top (Table 5).

Table 5. Comparison of tepal color among different populations.
\begin{tabular}{l|c|c}
\hline Cultivar & Abaxial tepal color & Adaxial tepal color \\
\hline JH1 & RHS 67A - RHS 67B & RHS 67B - RHS 67C \\
\hline JH2 & RHS 63B - RHS 63D & RHS 63B - RHS 63D \\
\hline JJ & RHS 62A - RHS 62B & RHS 62D \\
\hline JZ & RHS 62A - RHS 62B & Nearly white \\
\hline JY & RHS 63B - RHS 63C & RHS 62C - RHS 62D \\
\hline WS & RHS 62B - RHS 67A & Nearly white \\
\hline MD & White & White \\
\hline
\end{tabular}

$\mathrm{JH} 1=$ 'Jiaohong No.1', JH2 = 'Jiaohong No.2', JJ = 'Jiaoju', JZ= 'Jiaozi', JY = 'Jiaoyan'; reference populations: $\mathrm{WS}=$ Magnolia wufengensis wild species, $\mathrm{MD}=$ M. denudata .

The anthocyanin content was further measured using the HPLC method (Figure 2A). The result showed that the anthocyanin contents of $\mathrm{JH} 1$ and $\mathrm{JH} 2$ were significantly higher than those of the other group (Figure 2B). Among M. wufengensis cultivars, JJ was found to have the lowest pigment level. Compared with MD, whose anthocyanin content was barely detectable, populations of $M$. wufengensis possessed a significant abundance of floral pigments (Figure 2B).

Both RHSCC and HPLC methods confirmed a high level of anthocyanin content as well as a high variety for flower color in $M$. wufengensis cultivars. 


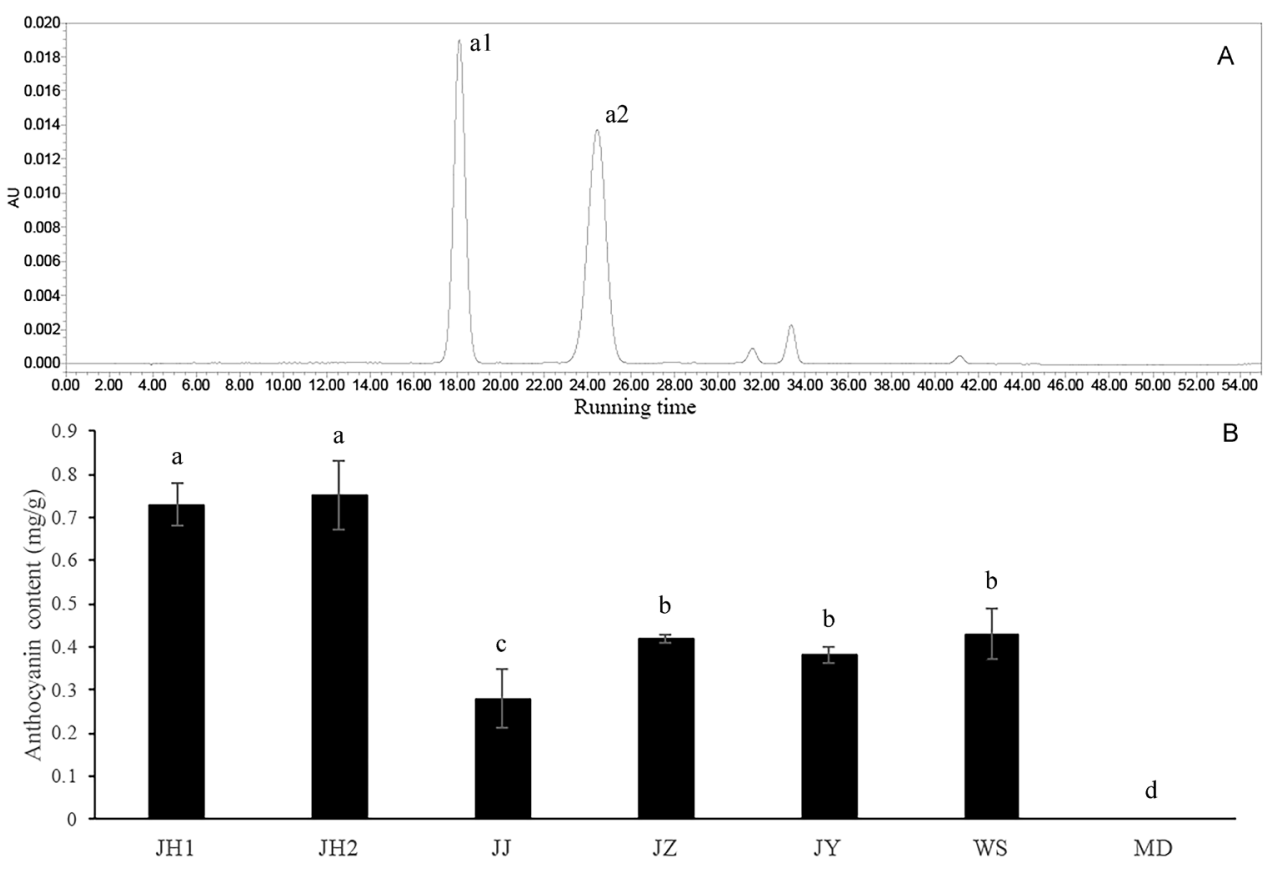

Figure 2. Anthocyanin content of Magnolia wufengensis cultivars. A. analysis of anthocyanin content using the HPLC method. The $\mathrm{x}$-axis and $\mathrm{y}$-axis indicate the running time and electric signal, respectively. Peaks a1 and a2 were assigned as anthocyanin; B. comparisons among M. wufengensis cultivars and reference species. Data are reported as means $\pm \mathrm{SD}$, different letters indicate a significant difference between different populations $(\mathrm{P}<0.05)$.

\section{SSR and SRAP characterizations}

The association of the SSR and SRAP primer characters, number of loci $(\mathrm{N})$, number of polymorphic loci $(\mathrm{Np})$, and percentage of polymorphic loci $(\mathrm{P})$ were evaluated using EXCEL (Table 6). There were nine efficient SSR primer pairs, which produced 48 loci, among which 43 loci were polymorphic. With an average of 5.33, N ranged from 2 (LT083) to 9 (LT078) per primer pair. The Np ranged from 1 to 8 for each primer pair, which resulted in a high P value ranging from 50\% (LT083) to 100\% (LT023), with $88.12 \%$ being the average. For SRAP, 10 efficient primer pairs were obtained and 170 loci were obtained with $93.53 \%$ of the $\mathrm{P}$ value (161 loci). For each primer, $\mathrm{N}$ ranged from 10 (em5-me5) to 23 (em1-me3), and Np ranged from 8 (em5-me5) to 22 (em6-me5). On average, SRAP was relatively more variable than SSR (N: 17, Np: 16, P: 92.89). Therefore, both of these molecular markers were highly polymorphic, but SRAP markers amplified more bands than SSR markers (Table 6).

\section{Genetic diversity analysis}

Four parameters were calculated for each population, including the percentage of polymorphic loci $(\mathrm{P})$, number of effective alleles $(\mathrm{Ne})$, mean expected heterozygosity $(\mathrm{H})$, and the Shannon index (I). On average, similar genetic diversity for $M$. wufengensis cultivars was observed when analyzed by SSR and SRAP $(0.341 \leq \mathrm{P} \leq 0.657 ; \alpha=0.05)$ (Table 7). 


\begin{tabular}{|c|c|c|c|c|c|c|c|}
\hline SSR primer & $\mathrm{N}$ & $\mathrm{Np}$ & $\mathrm{P}(\%)$ & SRAP primer & $\mathrm{N}$ & $\mathrm{Np}$ & $\mathrm{P}(\%)$ \\
\hline LT013 & 8 & 7 & 87.50 & em1-me3 & 23 & 21 & 91.30 \\
\hline LT023 & 5 & 5 & 100.00 & em1-me5 & 14 & 13 & 92.86 \\
\hline LT078 & 9 & 8 & 88.89 & em2-me 5 & 14 & 12 & 85.71 \\
\hline LT083 & 2 & 1 & 50.00 & em4-mel & 17 & 16 & 94.12 \\
\hline LT091 & 5 & 5 & 100.00 & em5-me3 & 15 & 15 & 100.00 \\
\hline LT092 & 3 & 3 & 100.00 & em5-me4 & 19 & 17 & 89.47 \\
\hline LT115 & 6 & 5 & 83.33 & em5-me5 & 10 & 8 & 80.00 \\
\hline LT149 & 6 & 5 & 83.33 & em5-me8 & 20 & 20 & 100.00 \\
\hline LT164 & 4 & 4 & 100.00 & em6-me5 & 22 & 21 & 95.45 \\
\hline- & - & - & - & eml-mel & 16 & 16 & 100.00 \\
\hline Mean & 5.33 & 4.78 & 88.12 & Mean & 17 & 16 & 92.89 \\
\hline
\end{tabular}

$\mathrm{N}$, number of loci; $\mathrm{Np}$, number of polymorphic loci; $\mathrm{P}$, percentage of polymorphic loci.

Table 7. Genetic diversity of Magnolia wufengensis cultivars analyzed by SSR and SRAP markers.

\begin{tabular}{l|c|c|c|c|c|c|c|c}
\hline \multirow{2}{*}{ Population } & \multicolumn{3}{|c|}{ SSR } & \multicolumn{3}{c}{ SRAP } \\
\cline { 2 - 9 } & $\mathrm{P}$ & $N_{\mathrm{E}}$ & $\mathrm{H}$ & $\mathrm{I}$ & $\mathrm{P}$ & $N_{\mathrm{E}}$ & $\mathrm{H}$ & $\mathrm{I}$ \\
\hline JH1 & $56.25 \%$ & 1.209 & 0.135 & 0.216 & $47.06 \%$ & 1.246 & 0.145 & 0.221 \\
\hline JH2 & $52.08 \%$ & 1.226 & 0.142 & 0.222 & $56.47 \%$ & 1.283 & 0.170 & 0.262 \\
\hline JJ & $37.50 \%$ & 1.232 & 0.134 & 0.199 & $41.76 \%$ & 1.293 & 0.162 & 0.237 \\
\hline JZ & $77.08 \%$ & 1.497 & 0.285 & 0.421 & $57.06 \%$ & 1.316 & 0.185 & 0.278 \\
\hline JY & $70.83 \%$ & 1.385 & 0.224 & 0.339 & $52.94 \%$ & 1.278 & 0.166 & 0.253 \\
\hline Mean & $58.75 \%$ & 1.310 & 0.184 & 0.279 & $51.06 \%$ & 1.283 & 0.166 & 0.250 \\
\hline Total & $89.85 \%$ & 1.896 & 0.289 & 0.437 & $89.41 \%$ & 1.894 & 0.286 & 0.432 \\
\hline WS & $81.25 \%$ & 1.491 & 0.287 & 0.429 & $78.24 \%$ & 1.447 & 0.256 & 0.400 \\
\hline MD & $43.75 \%$ & 1.278 & 0.164 & 0.243 & $38.24 \%$ & 1.259 & 0.147 & 0.216 \\
\hline
\end{tabular}

SSR, simple sequence repeat, SRAP, sequence-related amplified polymorphism; P, percentage of polymorphic loci; $N_{\mathrm{E}}$, number of effective alleles; H, expected heterozygosity; I, Shannon index.

For SSRs, an average $58.75 \%$ of polymorphic loci were amplified within $M$. wufengensis cultivars, among which the population JZ was found to possess a significantly high level of genetic diversity $(\mathrm{P}<0.01)$, while the population JJ showed slightly lower genetic variation. Compared to an outer reference species, populations JZ and JY had significantly higher values for genetic diversity $(\mathrm{P}<0.05)$. The SRAP marker system could amplify an average $51.06 \%$ polymorphic loci for $M$. wufengensis cultivars, which was similar to the value obtained for SSR. In accordance with the results obtained using SSR markers, the population JZ showed higher genetic diversity and JJ had lower variation. When compared with the outer species population, all the cultivar populations showed a significant increase in genetic diversity $(\mathrm{P}>0.1)$. Both the marker systems indicated that the wild species of $M$. wufengensis had the highest genetic diversity among all populations, and the five cultivars were found to possess significantly lower levels of genetic diversity than wild species $(\mathrm{P}<0.01)$ (Table 7). Both these marker systems confirmed that the five cultivars as a whole obtained the highest genetic diversity.

\section{Genetic distance analysis}

The genetic identity matrix was constructed by Nei's original measures of genetic identity and genetic distance (Nei, 1972). The pair-grouped genetic distances (D) among all seven populations for SSR varied from 0.0217 to 0.3566 , and among $M$. wufengensis cultivars 
were relatively narrower in range, from 0.0602 to 0.1604 (Table 8). For SSRs, the largest distance occurred between MD and $\mathrm{JH} 2$, and the smallest distance was between $\mathrm{JH} 2$ and wild species. The largest distance between $M$. wufengensis varieties was found between $\mathrm{JJ}$ and JH2, and the smallest distance was between JZ and JY. For SRAP, the pairwise distances varied from 0.0842 to 0.3325 , with the largest distance occurring between JH1 and MD, and the smallest distance between JH1 and JZ. Among M. wufengensis cultivars, the largest genetic distances were slightly less, at 0.2672 , which was observed between JH1 and JJ. In both SSR and SRAP, wild species was indicated to be much closer to cultivars $(\mathrm{D}<0.1210)$ than to outer species MD (D > 0.1867) (Table 8).

Table 8. Genetic distance analyses using SSR and SRAP markers.

\begin{tabular}{l|c|c|c|c|c|c|c}
\hline Pop ID & JH1 & JH2 & JJ & JZ & JY & WS & MD \\
\hline JH1 & - & 0.2459 & 0.2672 & 0.0842 & 0.1382 & 0.1175 & 0.3325 \\
\hline JH2 & 0.0861 & - & 0.2463 & 0.2078 & 0.1959 & 0.0918 & 0.2641 \\
\hline JJ & 0.1085 & 0.1604 & - & 0.1886 & 0.2203 & 0.1210 & 0.2641 \\
\hline JZ & 0.0660 & 0.1385 & 0.0996 & - & 0.1186 & 0.1005 & 0.2512 \\
\hline JY & 0.0901 & 0.1144 & 0.1332 & 0.0602 & - & 0.1196 & 0.2901 \\
\hline WS & 0.0637 & 0.0217 & 0.0531 & 0.0881 & 0.0908 & - & 0.2048 \\
\hline MD & 0.3368 & 0.3566 & 0.3405 & 0.2383 & 0.1867 & 0.3278 & - \\
\hline
\end{tabular}

Figures above the diagonal (marked by dashes) represent Nei's genetic distance detected by SRAP markers and below the diagonal are Nei's genetic distance detected by SSR markers. Pop ID: JH1 = M. wufengensis 'Jiaohong No.1', JH2 = M. wufengensis 'Jiaohong No.2', JJ $=$ M. wufengensis 'Jiaoju', JZ $=$ M. wufengensis 'Jiaozi', JY $=M$. wufengensis 'Jiaoyan', WS $=M$. wufengensis wild species, $\mathrm{MD}=M$. denudata.

\section{Analysis of genetic differentiation}

To study the genetic differentiation of $M$. wufengensis cultivars, AMOVA was performed within/among five $M$. wufengensis cultivars. Similar results were obtained with SSR and SRAP markers. SSR revealed that genetic variation within populations accounted for $70 \%$ of the total variation, while the variation among populations was $30 \%$. The $F_{\mathrm{ST}}$ value was 0.296 and indicated a high level of genetic differentiation. Similarly, with the SRAP markers, $80 \%$ of variation occurred within populations, and $20 \%$ occurred among populations. The $F_{\mathrm{ST}}$ assessed by SRAP was 0.197, indicating a moderate level of genetic differentiation (Table 9).

Table 9. Analyses of molecular variance (AMOVA) within/among five Magnolia wufengensis cultivars.

\begin{tabular}{l|l|c|c|c|c|c|c|c|c}
\hline & Source of variation & d.f. & SSD & MS & Est. Var. & Total variance & $F_{\text {ST }}$ & P value \\
\hline \multirow{3}{*}{ SSR } & Among population & 4 & 441.52 & 110.38 & 3.23 & $30 \%$ & 0.296 & $* * *$ \\
\cline { 2 - 10 } & Within population & 154 & 1184.12 & 7.69 & 7.69 & $70 \%$ & & \\
\cline { 2 - 10 } & Total & 158 & 1625.64 & & 10.92 & $100 \%$ & & \\
\hline \multirow{3}{*}{ SRAP } & Among population & 4 & 952.43 & 238.11 & 9.89 & $20 \%$ & 0.197 & $* * *$ \\
\cline { 2 - 9 } & Within population & 95 & 3836.20 & 40.38 & 40.38 & $80 \%$ & & \\
\cline { 2 - 9 } & Total & 99 & 4788.63 & & 50.27 & $100 \%$ & & \\
\hline
\end{tabular}

d.f., degrees of freedom; SSD, sum of squared deviations; MS, estimated variation; Est. Var., fixation index; $F_{\mathrm{ST}}$, the probability of having a more extreme variance component than the observed values by chance alone; $* * *$ significant at $\mathrm{P} \leq 0.001$.

\section{Clustering analysis}

Based on the phenotypic traits of floral organs (Table 4 and Figure 2B), clustering 
analysis was performed on the seven populations (Figure 3A). Unexpectedly, JJ was distantly separated from all other M. wufengensis populations. With the exception of JJ, all the other cultivars clustered together, and grouped with WS as one subgroup, which was clearly separated from the outer species, MD.

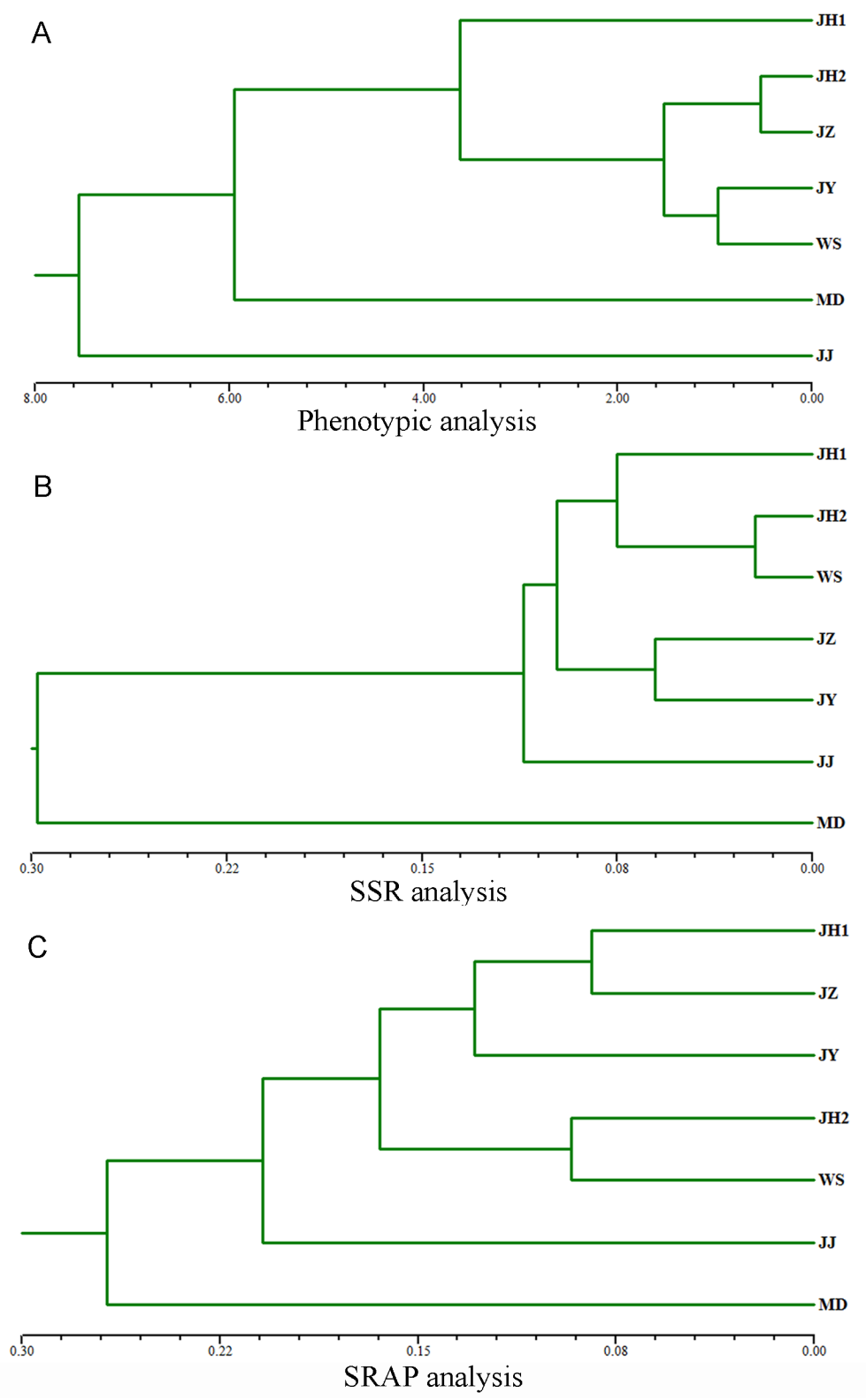

Figure 3. Clustering analyses of seven populations through phenotypic traits, SSR, and SRAP markers. Tree A was built using phenotypic traits; Tree $\mathbf{B}$ was built using SSR data; Tree $\mathbf{C}$ was built using SRAP data. Bootstrap values are indicated as $5 \%$ of 1000 replicates.

Genetics and Molecular Research 16 (1): gmr16019473 
Based on the identity matrix, the UPGMA dendrogram generated using both SSR and SRAP markers indicated that the first major group was divided into two sub clusters as shown in Figure $3 \mathrm{~B}$ and $3 \mathrm{C}$. One sub cluster was the reference outer species group $\mathrm{MD}$, and the other subgroup was the $M$. wufengensis group, which included all five new cultivars and the wild species. The dendrograms generated using SSR and SRAP showed that the M. wufengensis group further clustered into two main subgroups as shown in Figure 3B, C, one was comprised of JH1, JH2, JY, JZ, and the WS, while JJ alone formed the other subgroup. The markers differed slightly in the first subgroup. With SSRs, the population WS was closest to JH2, and then grouped successively with JH1, JY, and JZ (Figure 3B). With SRAPs, the population WS was closest to JH2. JH1, and JZ clustered as a sub branch, which firstly clustered with JY then with JH2 group (with WS) (Figure 3C).

\section{Correlation between phenotypic traits, SSR, and SRAP markers}

The Mantel correlation was moderate between genetic and geographical distances $(\mathrm{r}=$ $0.52, \mathrm{P}<0.01)$ for SSR and for SRAP $(\mathrm{r}=0.60, \mathrm{P}<0.01)$ (Figure 4A-C).

The correlation between SSR and SRAP markers was determined by analysis between $\mathrm{P}, \mathrm{H}, N_{\mathrm{E}}$, and I of the same cultivars, and the Mantel test of the two genetic distance matrices. The correlation coefficients of P, H, $N_{\mathrm{E}}$, and I between SSR and SRAP markers were 0.70, $0.71,0.72$, and 0.71 respectively, which inferred a relatively high correlation between the two markers. Meanwhile, the correlation coefficient evaluated by the Mantel test of the two genetic distance matrices was $0.73(\mathrm{P}<0.01)$ (Figure 4C), which also indicated a good fit of the two markers.
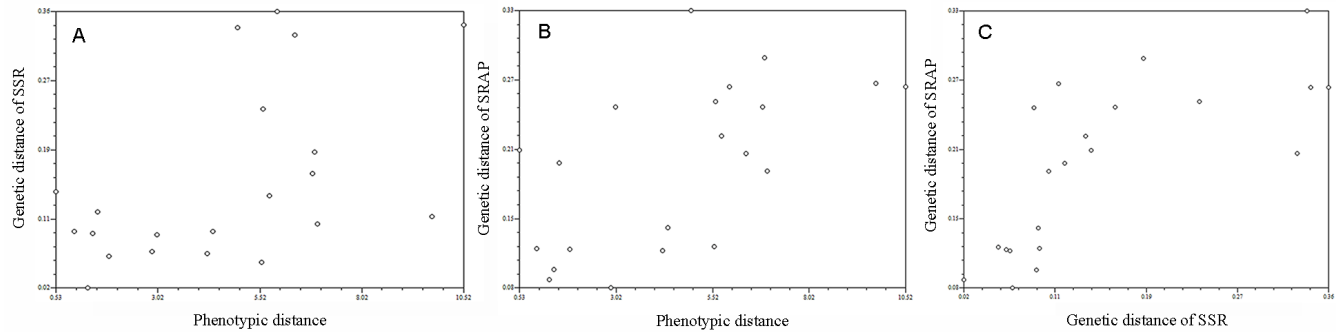

Figure 4. Consistence Mantel test for SSR and SRAP data and the correlation between genetic and phenotypic distance. A. Correlation between population genetic distance for SSR data and phenotypic distance; B. correlation between population genetic distance for SRAP data and phenotypic distance; C. correlation between SSR and SRAP data.

\section{DISCUSSION}

\section{Analysis of $M$. wufengensis cultivars and their relationship with other populations}

The utilization of predominant morphological traits could provide the most straightforward descriptions for further classification. Since M. wufengensis was found to be highly diversified in phenotypes of different organs, the morphological traits were used as the first reference to discriminate the cultivars and acted as the basis of genetic identification.

Genetics and Molecular Research 16 (1): gmr16019473 
In this study, we mainly investigated the characteristics of floral organs, and ultimately, six traits were screened. With the ability to determine the high variation of floral phenotypes, these traits were informative to identify $M$. wufengensis cultivars. However, considering the incorrect clustering of $\mathrm{JJ}$, such limited traits were not adequately precise to determine the relationship between cultivars. In addition, some traits had limitations that would make cultivars difficult to distinguish, for example, young seedlings bloom after 3-4 years of first propagation. Therefore, morphology represents the first step and should be followed by genetic analyses based on molecular markers.

The SSR and SRAP profiles were complementary for the identification of $M$. wufengensis cultivars. To comprehensively evaluate the genetic diversity of these cultivars, the parameters $\mathrm{P}, \mathrm{H}, N_{\mathrm{E}}$, and I were calculated. Compared with Magnolia officinalis cultivars (He et al., 2009), and cultivars of tree plant species like Olea europaea and tree peony (Casas et al., 2014; Yuan et al., 2014), these five M. wufengensis cultivars overall possess high genetic variation, but had relatively low genetic diversity for each individual cultivar. Interestingly, both SSR and SRAP markers revealed that the genetic diversity of population JJ was much lower than the average genetic diversity (which was already relatively low). Linked with its distinct phenotypic traits, cultivar JJ might be a valuable material for further study on floral organ development. SSR and SRAP analysis show diverse results with regard to the genetic diversity of cultivars JZ and JY. SSR analysis showed that variation in these two cultivars was moderate, while SRAP analysis showed low diversity.

The Mantel test failed to obtain a good-fit between phenotypic traits and molecular markers. This can be explained by the limited number of phenotypic traits, which may overlook differences in other organs and lead to inaccurate analysis, and also because the molecular markers were not being specifically developed for M. wufengensis, nor were they functionally linked. Thus, it was necessary to conduct further studies, including investigation of more observable morphological traits, development of more markers, and the use of other marker systems to precisely evaluate the genetic background.

In addition, the mean within-population genetic diversity for wild species of $M$. wufengensis was similar to the data reported in previous ISSR and SRAP analyses (Chen et al., 2014). However, for cultivars, the parameter values within-population of $M$. wufengensis were much lower. Similar results on the difference between cultivars and wild species have been obtained in previous studies. Either genetically related species such as M. officinalis and Magnolia cathcartii, or other economically important species such as Siraitia grosvenorii, were shown to have lower gene variation within cultivars than within wild types (Tang et al., 2007; He et al., 2009; Zhang et al., 2010).

The lower diversity may be attributed to the reproductive pattern. It has been reported that the mean within-population genetic diversities $(\mathrm{H})$ among different pollination systems (self-pollinated, mixed-mating, and outcrosses) were $0.120,0.180$, and 0.250 , respectively (Nybom, 2004). This implied that $M$. wufengensis cultivars probably had a mixed-mating system.

Additionally, since $M$. wufengensis is an endemic species with small habitat area and population size, developing a new cultivar and increasing its population in a short term by propagating from seed is apparently insufficient. Thus, clonal propagation e.g., grafting, has been widely performed in breeding work, and might lead to the loss of genetic diversity. According to the results obtained from both SSR and SRAP analyse, the genetic diversities of M. wufengensis cultivars as well as its wild population were all higher than outer species.

Genetics and Molecular Research 16 (1): gmr16019473 
Since high genetic diversity frequently leads to a variety of breeding materials, this result implied that $M$. wufengensis gained an advantage by acting as a genetic breeding resource and is thus worthy of further development.

The cophenetic correlation between the tree and genetic distance matrix was found to be high $(r=0.92 ; \mathrm{P}<0.01)$, suggesting that cluster analysis could represent the similarity matrix well. In accordance with previous phylogeny research on this species (He et al., 2007), M. wufengensis populations were clearly separated from other species, which supported the fact that M. wufengensis is a distinct new species of Magnoliaceae. The genetic distances among cultivars implied a relatively close relationship between them $(\mathrm{D}<0.35)$, which were similar to the characteristics of some endemic species (Haque et al., 2010).

The close relationship between wild species and cultivars was confirmed by both marker systems, while in the cluster analyses, they could not be clearly separated, which contradicted the findings of studies on other planted tree species e.g., Malus (Zhang et al., 2012). This is because these cultivars originated from the wild species, which had a limited population, and no outer species were included in the breeding process. Furthermore, with only a few years of cultivation, there was not sufficient time for genetic isolation driven by human selection or genetic drift to occur. Small genetic distances among cultivars resulted in a slight difference between the two dendrogram trees analyzed by SSR and SRAP markers. Since the cultivars all belong to $M$. wufengensis, such small distances are reasonable.

$F_{\mathrm{ST}}=0.25$ means there was great differentiation between subpopulations; the range 0.15 to 0.25 indicates a moderate level of differentiation, while differentiation is negligible if $F_{\mathrm{ST}}$ is 0.05 or less (Wright, 1978). Given that $F_{\mathrm{ST}}$ values of $M$. wufengensis cultivars were no less than 0.197 , which was much higher than found in a previous study of wild species populations $\left(F_{\mathrm{ST}}=0.170\right)$ (Chen et al., 2014). Factors such as geographical isolation, population fragmentation, breeding system, and genetic drift are reported to be responsible for the high genetic differentiation (Hogbin and Peakall, 1999). Moreover, in small populations, genetic drift could clearly increase the differentiation (Hogbin and Peakall, 1999). Variation among populations increased markedly to $30 \%$, reflecting the genetic isolation by artificial selection. Therefore, disruption of gene flow might be another major cause.

\section{Validity of SSR and SRAP markers}

In this study, SSR and SRAP markers, two co-dominant markers, were chosen to evaluate the genetic diversity of $M$. wufengensis cultivars. Both of the these systems were highly informative. This result is close to the that found previously in wild $M$. wufengensis species through ISSR and AFLP (87.70 and 95.51\%, respectively) (He et al., 2007; Chen et al., 2014). Furthermore, based on the results of UPGMA dendrogram analyses, both of the systems could clearly separate the $M$. wufengensis cultivars from other closely related species. In addition, the population relationship structures were similar to those reported in our previous study (He et al., 2007). Thus we consider SSR and SRAP marker systems to have tremendous potential for the efficient identification of the genetic background of $M$. wufengensis cultivars.

Studies that have discussed the relationship between different marker systems have varied substantially (Powell et al., 1996). In the present study, the correlation analyses using the Mantel test on the two distance matrices indicated a relatively good fit between SSR and SRAP markers. By comparing the genetic diversity parameters of cultivars, similar cophenetic

Genetics and Molecular Research 16 (1): gmr16019473 
correlations were obtained. This result was supported by those of previous studies. For instance, in a comparative analysis of lotus genetic diversity, a good correlation was found between SSR and SRAP markers $(r=0.732)$ (Yang et al., 2012b); however, this was lower than that observed in a study on lemons, where the cophenetic correlation between SSR and SRA reached as high as 0.97 (Uzun et al., 2011). This difference between the two markers may result from the limited number of efficient primer pairs, especially for the SSR markers. A SSR database for $M$. wufengensis would increase the number of primers, and a higher coefficient might also be obtained.

Since the results of the two markers were highly correlated in our study, analyses on the genetic diversity of $M$. wufengensis cultivars proved to be reliable.

\section{Insights into breeding and conservation}

The need for new cultivars is an ongoing process that is dependent on knowledge of the theoretical basis and progress of cultivation techniques. Phenotypic diversity was the basis for selection and subsequent breeding of new cultivars for the developing cultivation systems (Lavee, 2011). In the present study, we monitored the morphological phenotype of floral organs, which showed the most identical variations, implying that these have the potential to become breeding selection standards. Through the accurate description of the phenotype of each cultivar, we could establish the most direct classification system.

Given that morphological traits might occasionally be unreliable or limited, especially when the biotope changes (Nicotra et al., 2010), conservation of diverse genetic resources becomes a major issue in the effort of breeding cultivars. For some traditionally planted crops, such as grapes, a mass of cultivars and little knowledge about their genetic background would make breeding work risky (Guo et al., 2012). In this study, two simple systems, SSR and SRAP, proved to be efficient. Therefore, phenotypic evaluation complemented with a genetic marker system could clearly differentiate between cultivars. For modern targeted breeding, more accurate markers linked with certain phenotype traits are required. Molecular methods, including SNPs or SSRs as used in this study, based on analogous gene expression in model plants and previously selected crossing series of crossings would be useful (Lavee, 2011). Since the continuous study of marker technologies is practical, rapid advances in $M$. wufengensis breeding may be expected in the near future.

There are only approximately 2000 individuals of $M$. wufengensis extant in the secondary forests at elevations of 1500-2000 $\mathrm{m}$ in Wufeng, Hubei Province. In fact, the occurrence of wild populations of $M$. wufengensis species is subject to decline along with the number of individuals. An effective and authentic tool, such as the International Union for Conservation of Nature (IUCN) criteria, to evaluate conservation status, would be practical for endemic and rare species (Turchetto et al., 2016). Levels of genetic diversity will affect the ability of the species to adapt to changes in their habitat, thus acquiring a good knowledge of genetic diversity levels and distribution is essential for conservation strategies (Wanjala et al., 2013). In this study, the cultivars were found to exhibit a lower level of genetic diversity; however, when analyzed as a whole, they were able to maintain a high level of genetic diversity. These results may strongly encourage the development of novel M. wufengensis cultivars. For species conservation, cultivars with more diverse genotypes would probably give rise to a high level genetic variation. Simultaneously, for each cultivar, one population with a similar genetic background would benefit from a screen for functionally linked genes for further artificial

Genetics and Molecular Research 16 (1): gmr16019473 
breeding. Currently, we are developing novel cultivars not only for ornamental purpose, but also for better stress tolerance (Yang et al., 2015b); such efforts might facilitate the detection of resistance-related genes and contribute to expanding the planting areas of the species. Furthermore, it is notable that clonal propagation may prevent extinction but is basically an evolutionary dead-end. With further development of cultivars, seedling recruitment in small populations should be encouraged to introduce higher levels of gene flow, especially for those with low genetic variation. Since the new cultivars maintain the original optimum traits of $M$. wufengensis, the selection and breeding of cultivars have profound implications for conserving this rare and precious plant species.

In general, based on morphological classification, SSR and SRAP marker systems were shown to be valid and obtained similar results in the evaluation of $M$. wufengensis cultivars. Most cultivars were found to possess low levels of genetic diversity, but the five cultivars could maintain a high level of genetic variation at the species level. Genetic relationships among populations were primarily confirmed and the high genetic differentiation indicated increasing isolation between cultivars. These findings provide important information on $M$. wufengensis cultivars, and provide a basis for further study on breeding strategies and functional genetics, which would eventually enhance the efficiency of cultivar development as well as species conservation.

\section{Conflicts of interest}

The authors declare no conflict of interest.

\section{ACKNOWLEDGMENTS}

Research supported by the Special Fund for Forest Scientific Research in Public Welfare [\#201504704] and the Fundamental Research Funds for the Central Universities [\#BLYJ201506]. We thank Chen Zhu (College of Biological Sciences and Technology, Beijing Forestry University) and Xiaoqiang Gong (College of Forestry, Beijing Forestry University) for their assistance in revising the manuscript.

\section{REFERENCES}

Abid G, Mingeot D, Udupa SM, Muhovski Y, et al. (2015). Genetic relationship and diversity analysis of faba bean (Vicia faba L. var. Minor) genetic resources using morphological and microsatellite molecular Markers. Plant Mol. Biol. 33: 1755-1767. http://dx.doi.org/10.1007/s11105-015-0871-0

Budak H, Shearman RC, Parmaksiz I, Gaussoin RE, et al. (2004). Molecular characterization of Buffalograss germplasm using sequence-related amplified polymorphism markers. Theor. Appl. Genet. 108: 328-334. http://dx.doi. org/10.1007/s00122-003-1428-4

Casas GL, Scollo F, Distefano G, Continella A, et al. (2014). Molecular characterization of olive (Olea europaea L.) Sicilian cultivars using SSR markers. Biochem. Syst. Ecol. 57: 15-19. http://dx.doi.org/10.1016/j.bse.2014.07.010

Chen LN, Ma QG, Chen YK, Wang BQ, et al. (2014a). Identification of major walnut cultivars grown in China based on nut phenotypes and SSR markers. Sci. Hortic. (Amsterdam) 168: 240-248. http://dx.doi.org/10.1016/j. scienta.2014.02.004

Chen LY, Chen FJ, He SC and Ma LY (2014). High genetic diversity and small genetic variation among populations of Magnolia wufengensis (Magnoliaceae), revealed by ISSR and SRAP markers. Biochem. Syst. Ecol. 17: 268-274.

Fan J, Zhu W, Kang H, Ma H, et al. (2012). Flavonoid constituents and antioxidant capacity in flowers of different Zhongyuan tree penoy cultivars. J. Funct. Foods 4: 147-157. http://dx.doi.org/10.1016/j.jff.2011.09.006

Genetics and Molecular Research 16 (1): gmr16019473 
Guo D, Zhang J, Liu C, Zhang G, et al. (2012). Genetic variability and relationships between and within grape cultivated varieties and wild species based on SRAP markers. Tree Genet. Genomes 8: 789-800. http://dx.doi.org/10.1007/ $\underline{\text { s11295-011-0464-5 }}$

György Z, Vouillamoz JF and Höhn M (2016). Microsatellite markers reveal common East Alpine-Carpathian gene pool for the arctic-alpine Rhodiola rosea (Crassulaceae). Plant Syst. Evol. 302: 721-739. http://dx.doi.org/10.1007/ s00606-016-1302-x

Haque I, Bandopadhyay R and Mukhopadhyay K (2010). Population genetic structure of the endangered and endemic medicinal plant Commiphora wightii. Mol. Biol. Rep. 37: 847-854. http://dx.doi.org/10.1007/s11033-009-9661-9

He J, Chen L, Si Y, Huang B, et al. (2009). Population structure and genetic diversity distribution in wild and cultivated populations of the traditional Chinese medicinal plant Magnolia officinalis subsp. biloba (Magnoliaceae). Genetica 135: 233-243. http://dx.doi.org/10.1007/s10709-008-9272-8

He SC, Ma LY and Chen FJ (2007). Genetic diversity of Magnolia wufengensis based on AFLP. Acta Bot BorealiOccident. Sin. 27: 2421-2428.

He YQ, Ma LY and Sang ZY (2010). Preliminary Study on Formation of Flower Color in Magnolia wufengensis. Acta Bot. Boreali-Occident. Sin. 30: 2252-2257.

Hogbin PM and Peakall R (1999). Evaluation of the contribution of genetic research to the management of the endangered plant Zieria prostrata. Conserv. Biol. 13: 514-522. http://dx.doi.org/10.1046/j.1523-1739.1999.98182.x

Lavee S (2011). Genetic resources, from conservation to new cultivars. Acta Hortic. 924: 263-276. http://dx.doi. org/10.17660/ActaHortic.2011.924.33

Li G and Quiros CF (2001). Sequence-related amplified polymorphism (SRAP), a new marker system based on a simple PCR reaction: its application to mapping and gene tagging in Brassica. Theor. Appl. Genet. 103: 455-461. http:// dx.doi.org/10.1007/s001220100570

Ma LY, Wang LR, He SC, Liu X, et al. (2006). A new species of Magnolia (Magnoliaceae) from Hubei, China. Bull. Bot. Res. 26: 4-7.

Mratinić E, Akšić MF, Rakonjac V, Miletić R, et al. (2014). Morphological diversity of cornelian cherry (Cornus mas L.) populations in the Stara Planina Mountain, Serbia. Plant Syst. Evol. 301: 365-374. http://dx.doi.org/10.1007/ s00606-014-1079-8

Nei M (1972). Genetic distance between populations. Am. Nat. 106: 283-292. http://dx.doi.org/10.1086/282771

Nicotra AB, Atkin OK, Bonser SP, Davidson AM, et al. (2010). Plant phenotypic plasticity in a changing climate. Trends Plant Sci. 15: 684-692. http://dx.doi.org/10.1016/j.tplants.2010.09.008

Nybom H (2004). Comparison of different nuclear DNA markers for estimating intraspecific genetic diversity in plants. Mol. Ecol. 13: 1143-1155. http://dx.doi.org/10.1111/j.1365-294X.2004.02141.x

Powell W, Morgante M, Andre C, Hanafey M, et al. (1996). The comparison of RFLP, RAPD, AFLP and SSR (microsatellite) markers for germplasm analysis. Mol. Breed. 2: 225-238. http://dx.doi.org/10.1007/BF00564200

Sabir JS, Abo-Aba S, Bafeel S, Zari TA, et al. (2014). Characterization of ten date palm (Phoenix dactylifera L.) cultivars from Saudi Arabia using AFLP and ISSR markers. C. R. Biol. 337: 6-18. http://dx.doi.org/10.1016/j.crvi.2013.11.003

Souframanien J and Gopalakrishna T (2004). A comparative analysis of genetic diversity in blackgram genotypes using RAPD and ISSR markers. Theor. Appl. Genet. 109: 1687-1693. http://dx.doi.org/10.1007/s00122-004-1797-3

Tang N, Shahin A, Bijman P, Liu JJ, et al. (2013). Genetic diversity and structure in a collection of tulip cultivars assessed by SNP markers. Sci. Hortic. (Amsterdam) 161: 286-292. http://dx.doi.org/10.1016/j.scienta.2013.07.016

Tang SQ, Bin XY, Peng YT, Zhou JY, et al. (2007). Assessment of genetic diversity in cultivars and wild accessions of Luohanguo (Siraitia grosvenorii), a species with edible and medicinal sweet fruits endemic to southern China, using RAPD and AFLP markers. Genet. Resour. Crop Evol. 54: 1053-1061. http://dx.doi.org/10.1007/s10722-006-9190-5

Turchetto C, Segatto ALA, Mäder G, Rodrigues DM, et al. (2016). High levels of genetic diversity and population structure in an endemic and rare species: implications for conservation. AoB Plants 8: 8. http://dx.doi.org/10.1093/ aobpla/plw002

Uzun A, Yesiloglu T, Polat I, Aka-Kacar Y, et al. (2011). Evaluation of genetic diversity in lemons and some of their relatives based on SRAP and SSR markers. Plant Mol. Biol. Report. 29: 693-701. http://dx.doi.org/10.1007/s11105$\underline{010-0277-y}$

Wanjala BW, Obonyo M, Wachira FN, Muchugi A, et al. (2013). Genetic diversity in Napier grass (Pennisetum purpureum) cultivars: implications for breeding and conservation. AoB Plants 5: plt022. http://dx.doi.org/10.1093/aobpla/plt022

Wright S (1978). Evolution and the genetics of populations, Volume 4: Variability within and among natural populations. University of Chicago Press, Chicago.

Xu M, Sun YG and Li HG (2010). EST-SSRs development and paternity analysis for Liriodendron spp. New For. 40: 361-382. http://dx.doi.org/10.1007/s11056-010-9205-0

Genetics and Molecular Research 16 (1): gmr16019473 
Yang AH, Zhang JJ, Tian H and Yao XH (2012a). Characterization of 39 novel EST-SSR markers for Liriodendron tulipifera and cross-species amplification in L. chinense (Magnoliaceae). Am. J. Bot. 99: e460-e464. http://dx.doi. org/10.3732/ajb.1200154

Yang M, Han YN, Xu LM, Zhao JR, et al. (2012b). Comparative analysis of genetic diversity of lotus (Nelumbo) using SSR and SRAP markers. Sci. Hortic. (Amsterdam) 142: 185-195. http://dx.doi.org/10.1016/j.scienta.2012.05.021

Yang Y, Jia ZK, Chen FJ, Sang ZY, et al. (2015a). Natural cold acclimatisation and de-acclimatisation of Magnolia wufengensis in response to alternative methods of application of abscisic acid. J. Hortic. Sci. Biotechnol. 90: 704710. http://dx.doi.org/10.1080/14620316.2015.11668734

Yang Y, Jia ZK, Chen FJ, Sang ZY, et al. (2015b). Comparative analysis of natural cold acclimation and deacclimation of two Magnolia species with different winter hardiness. Acta Physiol. Plant. 37: 1-11. http://dx.doi.org/10.1007/ s11738-015-1883-y

Yang Y, Jia ZK, Chen FJ, Sang ZY, et al. (2015c). Physiological and biochemical processes of Magnolia wufengensis in response to foliar abscisic acid application during natural cold acclimation. HortScience 50: 387-394.

Yuan JH, Cornille A, Giraud T, Cheng FY, et al. (2014). Independent domestications of cultivated tree peonies from different wild peony species. Mol. Ecol. 23: 82-95. http://dx.doi.org/10.1111/mec.12567

Zhang Q, Li J, Zhao YB, Korban SS, et al. (2012). Evaluation of genetic diversity in Chinese wild apple species along with apple cultivars using SSR markers. Plant Mol. Biol. Report. 30: 539-546. http://dx.doi.org/10.1007/s11105$\underline{011-0366-6}$

Zhang XM, Wen J, Dao ZL, Motley TJ, et al. (2010). Genetic variation and conservation assessment of Chinese populations of Magnolia cathcartii (Magnoliaceae), a rare evergreen tree from the South-Central China hotspot in the Eastern Himalayas. J. Plant Res. 123: 321-331. http://dx.doi.org/10.1007/s10265-009-0278-9

Genetics and Molecular Research 16 (1): gmr16019473 